Discrete Comput Geom 26:387-410 (2001)

DOI: $10.1007 /$ s00454-001-0042-y

\title{
Every Set of Disjoint Line Segments Admits a Binary Tree*
}

\author{
P. Bose, ${ }^{1}$ M. E. Houle, ${ }^{2}$ and G. T. Toussaint ${ }^{3}$ \\ ${ }^{1}$ School of Computer Science, Carleton University, \\ Ottawa, Ontario, Canada K1S 5B6 \\ jit@scs.carleton.ca \\ 2 Basser Department of Computer Science, The University of Sydney, \\ Sydney, NSW 2006, Australia \\ meh@cs.usyd.edu.au \\ ${ }^{3}$ School of Computer Science, McGill University, \\ Montreal, Quebec, Canada H3A 2A7 \\ godfried@cs.mcgill.ca
}

\begin{abstract}
Given a set of $n$ disjoint line segments in the plane, we show that it is always possible to form a tree with the endpoints of the segments such that each line segment is an edge of the tree, the tree has no crossing edges, and the maximum vertex degree of the tree is 3. Furthermore, there exist configurations of line segments where any such tree requires degree 3. We provide an $O(n \log n)$ time algorithm for constructing such a tree, and show that this is optimal.
\end{abstract}

\section{Introduction}

Given a set of disjoint line segments, determining whether the set admits certain combinatorial structures has received considerable attention. One of the best-studied such structures has been the simple circuit or polygon through a set of line segments. The question of deciding whether a set of disjoint line segments admits a simple circuit is conjectured to be NP-complete, since Rappaport [12] has shown that deciding whether a set of line segments allowed to intersect at their endpoints admits a simple circuit is an NP-complete problem. For certain special cases, however, polynomial-time algorithms have been obtained. Avis and Rappaport [1] gave an $O\left(n^{4}\right)$ time and $O\left(n^{2}\right)$ space algorithm to decide whether a set of disjoint line segments admits a simple monotone

* The research by P. Bose was supported by NSERC. 
circuit. Rappaport et al. [13] have shown that the decision problem is in $O(n \log n)$ when every line segment in the set has at least one endpoint on their convex hull (such a configuration is known as a convexly independent set of line segments). Although not every convexly independent set of line segments admits a simple circuit, Mirzaian [7] has shown that such a set always admits a simple polygon such that the line segments are either part of the boundary of the polygon or form internal diagonals. Mirzaian's result does not hold for arbitrary sets of disjoint line segments, as was shown by Urabe and Watanabe [17], and later by Grünbaum [6], but it is conjectured that the result is true if the line segments are also allowed to form external diagonals of the polygon.

The simple circuit is not the only structure to have been investigated. ElGindy and Toussaint [5] have shown that every set of line segments can be triangulated. Later, O'Rourke and Rippel [10] proved the hamiltonicity of the visibility graph of certain restricted classes of line segments.

The structures with which this paper is concerned are trees that span a set of disjoint line segments such that each line segment is an edge of the tree and the tree has no crossing edges-such a tree will be referred to as an encompassing tree. The problem of determining whether a set of line segments admits an encompassing tree was first studied by Bose and Toussaint [3], who showed that a set of disjoint line segments always admits an encompassing tree, and that the encompassing tree of minimum total edge length has maximum degree 7. Subsequently, Rivera-Campo and Urrutia [14] proved that a disjoint set of line segments always admits an encompassing tree with maximum degree 4 .

A natural question to ask is: Given a set of disjoint line segments, is there always an encompassing tree with maximum degree less than 4? Figure 1 shows that there exist configurations that do not admit an encompassing tree with maximum degree 2. However, we show that a set of disjoint line segments always admits an encompassing tree with maximum degree 3 (a binary tree), and that such a tree can be computed in optimal $\Theta(n \log n)$ time.

The encompassing tree construction relies heavily on a convex subdivision of the plane induced by the set of line segments. The construction of the subdivision is discussed in Section 2, and the special structure of the subdivision is examined in Section 3. In Section 4 it is shown how the subdivision may be used to construct an encompassing tree

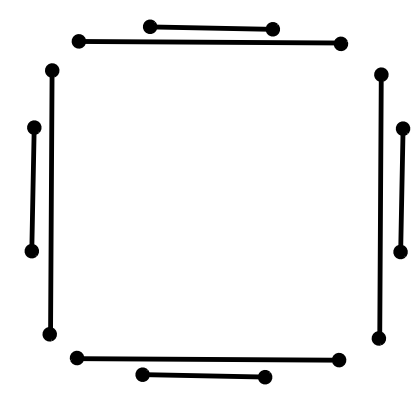

Fig. 1. No encompassing tree with maximum degree 2. 
of degree 3 in $O(n \log n)$ time. In Section 5 we present a proof of an $\Omega(n \log n)$ lower bound for the problem. Closing remarks and open problems can be found in Section 6 .

Most of the geometric and graph theoretic terminology used in this paper is standard, and for definitions we refer the reader to [9], [2], and [11].

\section{The Convex Subdivision}

The goal of the next three sections is to develop an algorithm to construct an encompassing tree $G$ (as defined earlier) for a set of $n$ disjoint line segments $S$. To simplify the description of the algorithm, and to avoid degeneracies, we assume throughout the paper that

- no segment of $S$ is horizontal (that is, parallel to the $x$-axis),

- no three endpoints of segments of $S$ are collinear, and

- of the lines obtained by extending the segments to infinity in either direction, no three intersect in a common point.

The first of these assumptions is easily realized-if horizontal segments are present, a simple reorientation of the coordinate axes can be performed in $O(n)$ time. The second and third assumptions can be realized using a perturbation scheme; however, we do not address these issues here.

To arrive at an algorithm for computing a degree-3 encompassing tree of $S$, we first construct a convex subdivision derived from the segments of $S$. Instead of subdividing the entire plane, we find it convenient to place a bounding box around the set of line segments, and to subdivide the interior of the box into convex regions. In so doing, we ensure that the subdivision has no unbounded regions or edges.

Conceptually, the subdivision is obtained by extending each segment $s$ along the unique line containing it. The extensions take the form of two rays, one oriented "upwards" (increasing in $y$-coordinate) and the other oriented "downwards" (decreasing in $y$-coordinate). Each ray is allowed to continue until it intersects an obstacle or another ray, at which point it is possibly truncated.

The rules governing these intersections are as follows:

1. If the intersection is determined by a ray $r$ and an edge $b$ of the bounding box, then $r$ is truncated at that intersection point: it does not continue beyond $b$.

2. If the intersection is determined by a ray $r$ and a segment $s$ of $S$, then $r$ is truncated at that intersection point: it does not continue beyond $s$.

3. If the intersection is determined by two rays $r_{1}$ and $r_{2}$ of the same orientation, then one ray is allowed to continue, and the other is truncated. We assume that $r_{2}$ intersects $r_{1}$ from the right (as viewed from $r_{1}$ ). If the rays are upward-oriented, then $r_{2}$ is truncated; if they are downward-oriented, $r_{1}$ is truncated.

4. If the intersection is determined by an upward-oriented ray $r_{\mathrm{u}}$ and a downwardoriented ray $r_{\mathrm{d}}$, then $r_{\mathrm{u}}$ is allowed to continue, and $r_{\mathrm{d}}$ is truncated.

See Fig. 2 for illustrations of each of these cases. 


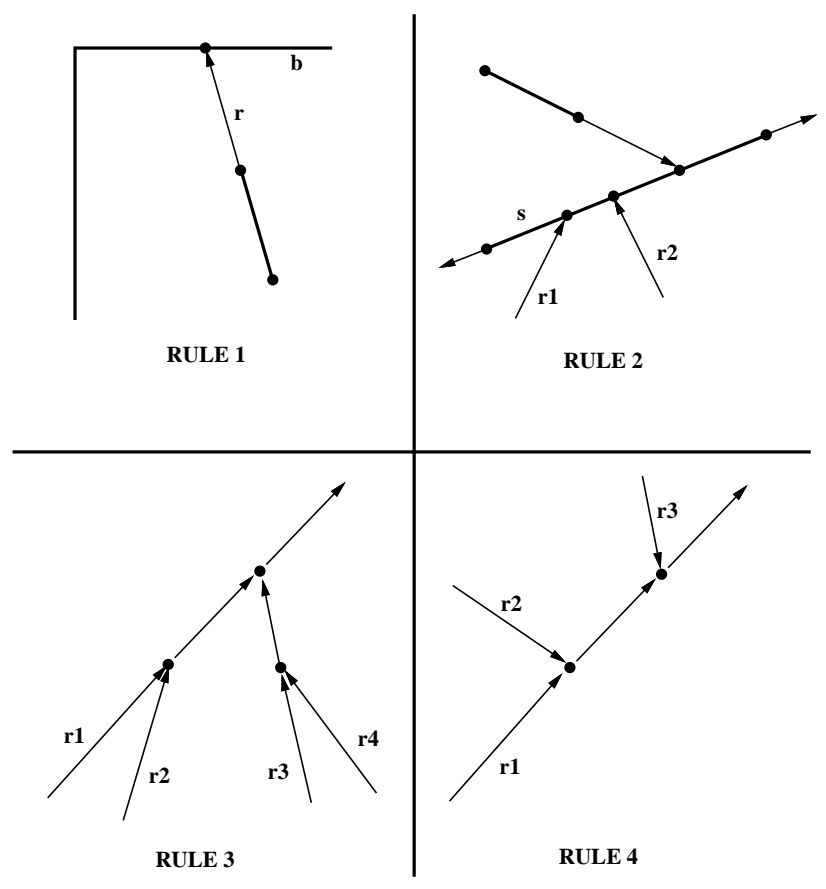

Fig. 2. Extension ray intersection rules.

These rules are sufficient to guarantee that the resulting subdivision is convex. A vertex $v$ of the subdivision is either an endpoint of a segment of $S$, a corner of the bounding box, or the truncation point of some ray, but in each of these cases, every angle incident to $v$ (and interior to the box) is at most $\pi$ by the construction. Thus every region is a polygon with no interior angle greater than $\pi$, and is thereby convex.

To construct the subdivision in an efficient manner, we make use of the well-known sweep-line paradigm. We assume that the reader is generally familiar with this paradigm, and present only a sketch of the construction here. For more information regarding sweepline techniques, see [11].

The sweep is done in two passes: in the first pass a horizontal line is swept from bottom to top, searching for intersections involving upward-oriented rays only-downwardoriented rays are ignored. When an intersection is detected, the appropriate rule $(1,2$, or 3 ) is applied.

In the second pass the downward-oriented rays are introduced. A horizontal line is swept from top to bottom, searching for intersections involving downward-oriented rays. When an intersection is detected, the appropriate rule $(1,2,3$, or 4$)$ is applied. Note that the fourth rule guarantees that the subdivision edges introduced in the first pass are not disturbed, as these edges derive from upward-oriented rays.

Consider the set of line segments (and its bounding box) shown in Fig. 3(a). The subdivisions obtained after the first and second passes are shown in Fig. 3(b) and (c), respectively. 


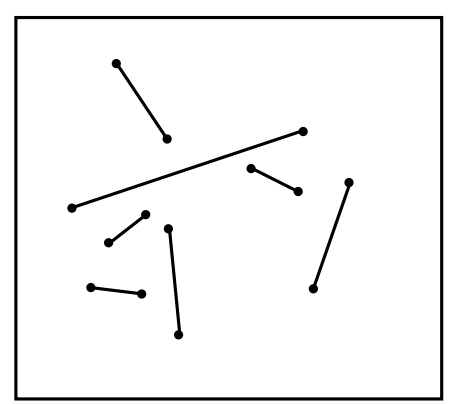

(a) Before

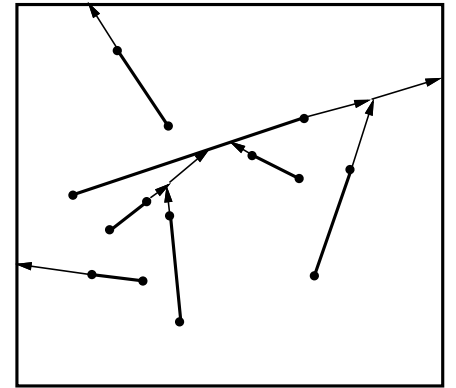

(b) After first pass

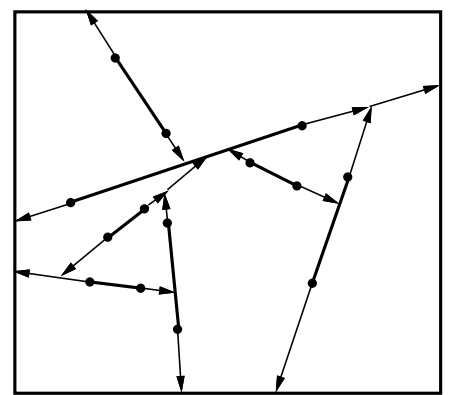

(c) After second pass

Fig. 3. Constructing the convex subdivision.

\section{Properties of the Convex Subdivision}

In this section we state and prove a number of facts concerning convex subdivisions of the kind described in the previous section. We also examine structures to be found within the subdivision which are central to the description of the algorithm presented in the following section, both in its motivation and in the proof of its correctness.

We assume throughout that $\mathrm{Q}$ is the subdivision for a set $S$ of $n$ line segments in the plane.

Lemma 1. The number of edges, vertices, and regions of $Q$ is in $O(n)$.

Proof. Follows easily from Euler's formula [2].

The edges of the subdivision can be of one of three types:

- segment edges, which derive from segments of $S$,

- extension edges, which derive from extension rays of segments of $S$, and

- box edges, which derive from the sides of the bounding box.

Each extension edge can be thought to have an orientation, namely that of the ray from which the edge is derived. It can be classified as an upward extension edge or a downward extension edge, depending on the orientation of the ray. 
Lemma 2. Every cycle in $Q$ (other than the cycle forming the bounding box) contains an endpoint of some segment in $S$.

Proof. Assume otherwise: that is, there exists some cycle $\eta$ that does not consist entirely of box edges, and that does not contain an endpoint of any segment in $S$. Note that the cycle must contain at least one extension edge.

Let $\eta^{\prime}=\left\{e_{0}, e_{1}, e_{2}, \ldots, e_{k-1}, e_{k}\right\}$ be the subsequence of $\eta$ consisting of the extension edges of $\eta$, where $e_{0}=e_{k}$. With respect to the ordering of $\eta^{\prime}$, each extension edge is oriented either forward or backward.

- Case I: the edges of $\eta^{\prime}$ do not all share the same orientation.

In this case there must exist some $i$ such that $e_{i}$ is backward and $e_{i+1}$ is forward. Clearly, $e_{i}$ and $e_{i+1}$ cannot share a common endpoint—otherwise, two rays would emanate from one point, in contravention of the rules governing intersections ( 3 and 4). This implies that there must be at least one non-extension edge between $e_{i}$ and $e_{i+1}$ in $\eta$. Let $e$ be the non-extension edge occurring immediately before $e_{i+1}$ in $\eta$.

Let $v$ be the vertex of $\mathrm{Q}$ where $e$ meets $e_{i+1}$. Vertex $v$ cannot lie on a box edge, since no extension ray can emanate from the side of the bounding box. Therefore $e$ must be a segment edge. However, $v$ must then be an endpoint of the underlying segment $s$ in $S$, since no extension ray can emanate from the side of $s$. This contradicts the assumption.

- Case II: the edges of $\eta^{\prime}$ all share the same orientation.

Without loss of generality, we can assume that the extension edges are all forward edges. The arguments of Case I imply that the cycle must consist entirely of extension edges-that is, $\eta=\eta^{\prime}$, and the cycle is the sequence $\eta$ itself. The edges of $\eta^{\prime}$ therefore cannot be all upward; otherwise, each vertex in the cycle would have the $y$-coordinate strictly greater than its predecessor, which is impossible. Similarly, the edges of $\eta^{\prime}$ cannot be all downward. Therefore there exists some $j$ such that $e_{j}$ is upward and $e_{j+1}$ is downward.

Let $v^{\prime}$ be the vertex where $e_{j}$ meets $e_{j+1}$. The edge $e_{j+1}$, being forward, is oriented away from $v^{\prime}$. Therefore $e_{j}$ (and not $e_{j+1}$ ) was on the ray that was truncated at $v^{\prime}$. However, this contravenes the fourth intersection rule of Section 2, by which the downward-oriented ray containing $e_{j+1}$ should have been truncated instead. Thus no simple cycle may have its extension edges share a common orientation.

Lemma 2 has an immediate implication concerning the structures formed by extension edges. Let $\mathrm{F}$ be the subgraph of $\mathrm{Q}$ induced by the extension edges of Q. Since Q cannot contain cycles consisting entirely of extension edges, $\mathrm{F}$ must be a forest; that is, each connected component of $\mathrm{F}$ is a tree. We refer to such trees as extension trees.

By the orientation of its incident edge, we can distinguish between two types of leaves of extension trees: those whose incident edges are directed away from the leaf, and those whose incident edges are directed towards the leaf. The former kind correspond to endpoints of segments of $S$; the latter kind can be formed only when an extension ray meets either the side of a segment or the bounding box. While an extension tree can have many leaves of the former kind, it turns out that it can have only one of the latter 
kind. We refer to these latter kinds of nodes as roots of their respective trees, reserving the term leaf for nodes of the former kind. The following lemma justifies the use of this terminology:

Lemma 3. If $T$ is an extension tree, then it has exactly one root. Furthermore, the edges along the path from any node to the root are all oriented towards the root.

Proof. According to the rules governing the intersections of extension rays, each internal node of the tree has exactly one outgoing edge. From any starting node $x$, we consider the set of nodes reachable from $x$ via a sequence of outgoing edges. Since $T$ has no cycles, and is finite, this sequence must describe a unique path in $T$ of finite length, oriented towards the terminus. Since the definition states that leaves are incident to outgoing edges, and roots to incoming edges, the terminus of this path can only be a root. This root is unique, since every internal node can have only one outgoing edge.

Even though the leaves of an extension tree may lie on many different segments of the subdivision, the uniqueness of the root allows us to associate each tree with either a unique segment of $S$, or the bounding box. Let $T_{1}$ and $T_{2}$ be extension trees rooted on the same side of a common segment $s$ of $S$, and let $r_{1}$ and $r_{2}$ be their respective roots. If no other extension tree rooted on the same side of $s$ has its root between $r_{1}$ and $r_{2}$, then we say that $T_{1}$ and $T_{2}$ are adjacent. In the same spirit, we say that two trees rooted on the bounding box are adjacent if it is possible to move along the bounding box from one root to the other without encountering the root of any other extension tree. See Fig. 4 for an example of adjacent extension trees.

Consider a segment $s$ of $S$, and the set $\mathcal{T}=\left\{T_{1}, T_{2}, \ldots, T_{k}\right\}$ of all trees rooted to one particular side of $s$. We assume that the trees of $\mathrm{T}$ are indexed in accordance with the left-to-right ordering of their roots with respect to $s$, as viewed towards $s$ from the side to which the trees attach. Let $\left(v_{1}, v_{2}, \ldots, v_{m}\right)$ be the sequence of leaves one would obtain if one reported them as they were encountered during an inorder traversal of all the trees of $\mathrm{T}$ in left-to-right order. With respect to this ordering, we say that $v_{i}$ is the

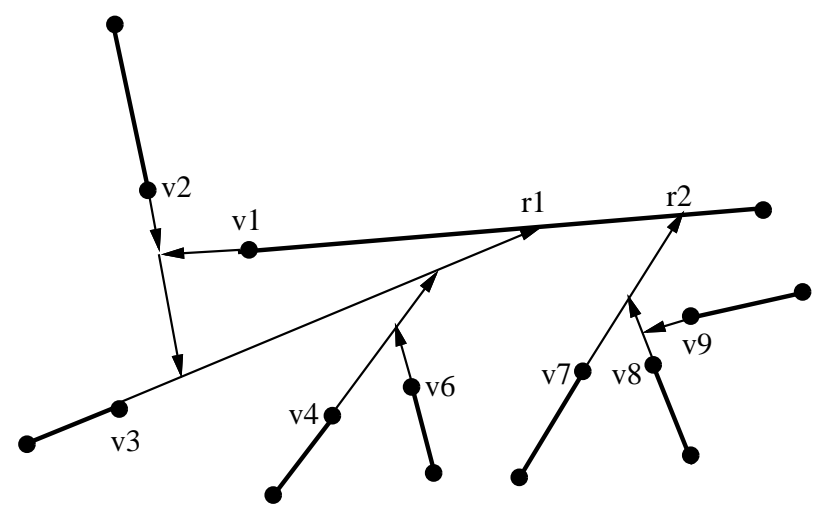

Fig. 4. Two adjacent extension trees. 
left neighbour of $v_{i+1}$, that $v_{i+1}$ is the right neighbour of $v_{i}$, and that $v_{i}$ and $v_{i+1}$ are neighbouring leaves (see Fig. 4).

Observation 4. Let $v$ and $w$ be neighbouring leaves with respect to some segment $s$ of $S$. Then there exists a path from $v$ to $w$ in $Q$ that

- passes only through extension edges of trees rooted at s, or segment edges contained in $s$, and

- that is entirely contained in the boundary of some cell c of $Q$.

Observation 5. Let $v$ be a leaf of an extension tree $T_{v}$ rooted at some segment $s$ of $S$. Let $s_{v}$ be the left endpoint of $s$ as viewed from the side to which the extension tree is rooted. If $v$ has no left neighbour, then there exists a path from $v$ to $s_{v}$ in $Q$ that

- passes only through extension edges of $T_{v}$, or segment edges contained in $s$, and

- that is entirely contained in the boundary of some cell c of $Q$.

Note that $v$ can be identical to $s_{v}$, in which case the extension tree of which $s_{v}$ is a leaf has its root at $s$. By symmetry, Observation 5 holds when $v$ has no right neighbour and $s_{v}$ is the right endpoint.

Observation 4 extends to the case where we consider all trees rooted at the bounding box. The only difference worth noting here is that whereas $v_{1}$ has no left neighbour and $v_{m}$ has no right neighbour in the case outlined above, every leaf of a tree rooted at the bounding box always has both a left and a right neighbour.

We conclude the discussion of the properties of the convex subdivision with the following lemma, that shows that all segments of $S$ can be connected simply by ensuring that for every cell $c$, the segments on the boundary of every cell $c$ are mutually connected.

Lemma 6. Let $S$ be a set of $n$ disjoint line segments, and let $Q$ be its underlying convex subdivision. Let $G$ be any planar graph whose vertex set is the set of endpoints of the segments in $S$ and whose edge set includes the segments of $S$. Then $G$ is connected if and only if for every cell $c$ of $Q$, the set of segment endpoints on the boundary of $c$ is connected in $G$.

Proof. If $G$ is connected, then trivially the set of segment endpoints on the boundary of any given cell are mutually reachable in $G$.

If the segment endpoints on the boundary of every cell $c$ are mutually connected in $G$, then the fact that $G$ is connect follows from Lemma 2 (i.e., every cycle in Q must contain an endpoint of $S$ ) and the fact that the planar dual [2] of Q is connected.

\section{Constructing an Encompassing Tree of Degree 3}

The degree-3 encompassing tree construction algorithm, ENCOMPASS, can best be described as incremental: starting from a single segment of $S$, previously unattached seg- 
ments are attached to a growing tree $G$ one by one until no unattached segments remain. When the algorithm terminates, $G$ is the encompassing tree for $S$.

In the next subsection we discuss some of the invariants and conventions observed by ENCOMPASS.

In Section 4.2 we present a key procedure of the overall algorithm, ATTACHTO-one which given a leaf of an extension tree, attaches to it the segment at which the tree is rooted.

Procedure ATTACHTO is not in itself sufficient to link up correctly all the segments into an encompassing tree of degree 3 . Although the main algorithm greedily relies on ATTACHTO to attach as many segments as possible to the growing connected component, it sometimes occurs that segments are left unattached even after all opportunities for applying ATTACHTO have been exhausted. In Section 4.3 we present the procedure STITCHUP that takes a cell with both attached and unattached segments in its boundary, and attaches to $G$ those segments that ATTACHTO could not find.

In Section 4.4 we present the main algorithm, as well as its complexity analysis, and a proof of correctness.

\subsection{Preliminaries}

Algorithm ENCOMPASS accepts as its input a set of segments $S$ and returns an encompassing tree $G$ of degree at most 3 . Whenever in the course of the execution of the algorithm an edge of $G$ is created between two segment endpoints $v$ and $w$, we say that a bridge $(v, w)$ has been created between $v$ and $w$.

The ENCOMPASS algorithm maintains the following invariants regarding the creation of bridges:

- A bridge is added only between two mutually visible endpoints.

- Each bridge added to the encompassing tree passes through the interior of exactly one cell of the subdivision Q, from one segment endpoint on its boundary to another segment endpoint on the boundary.

- Each endpoint can have at most two bridges attached to it, one through each of the two cells sharing the endpoint in their common boundary.

- A bridge is never created between two endpoints so as to introduce a cycle into $G$.

During the execution of the algorithm, as vertices are visited and bridges created, the segments, segment endpoints, and cells of $\mathrm{Q}$ acquire various labels. The labels also respect certain invariant conditions, outlined below.

A segment can be labeled unattached, in which case it has not yet been bridged to any other segment; attached, which indicates that it has been integrated into the final encompassing tree $G$; and semi-attached, which indicates that it has been connected to other segments by means of bridges, but has not yet been integrated into the final encompassing tree. Semi-attached segments are labeled with the name of a connected component into which it has been integrated. Initially, all segments are unattached. Once a segment becomes semi-attached, it will never again become unattached. Once it becomes attached, it will always remain attached. The bounding box as a whole will 
sometimes be treated as if it were a segment. It is initialized with the label unattached, and will eventually receive the label attached.

Segment endpoints can be labeled unvisited, pending, or examined. An endpoint is unvisited if its segment has not yet been attached to another. Otherwise, if it is a candidate leaf from which to apply ATTACHTO, then it carries the label pending. Endpoints labeled unvisited or pending have no bridges yet attached to them. An endpoint labeled examined is one from which a call to ATTACHTO is no longer necessary. Initially, all segment endpoints are unvisited. Once an endpoint becomes pending, it will never again become unvisited. Once it becomes examined, it will always remain examined.

The labels of the cells of Q depend on the labels of the segments having endpoints contained in its boundary. If these labels are all unattached, then the cell is labeled unvisited. If the segments are all attached, this implies that all endpoints in the boundary of the cell are mutually connected by the encompassing tree, and thus the cell acquires the label connected. A cell that is neither connected nor unvisited (that is, only "partly" connected) is labeled pending. Initially, all cells are unvisited. Once a cell becomes pending, it will never again become unvisited. Once it becomes connected, it will always remain connected. When all cells become connected, all segments are in $G$.

In the descriptions to come, the labels of cells are often not explicitly mentioned. We assume that every time a segment label is modified, the labels of the two cells upon which it borders are updated in accordance with the new segment label. This can be done simply by maintaining an appropriate counter for each cell.

\subsection{Connecting the Leaves of Extension Trees}

Under the assumption that no two segments are collinear, Observation 4 implies that subject to other restrictions (such as the invariants outlined in the previous subsection), a bridge can always be created between any two neighbouring leaves $v$ and $w$-unless $v$ and $w$ are opposite endpoints of the same segment of $S$, in which case no bridge is necessary. If endpoint $v$ has no left neighbour, then by Observation 5 a bridge can be created between $v$ and the left endpoint of the segment to which the extension tree of $v$ is rooted (and similarly if $v$ has no right neighbour). Algorithm ENCOMPASS takes advantage of this by means of its procedure ATTACHTO.

Procedure $\operatorname{ATtACHTO}(x$, dir $)$ accepts a leaf $x$ of an extension tree that is already contained in some connected component (that is, either semi-attached or attached), and a direction $\operatorname{dir}$ ("left" or "right"). If $T_{x}$ is the extension tree of which $x$ is a leaf, then the behaviour of ATTACHTO depends on whether $T_{x}$ is rooted at some segment $s^{*}$ of $S$, or at the bounding box. In the former case, ATTACHTO only proceeds if $s^{*}$ is unattached by traversing the trees rooted at $s^{*}$ towards one of the endpoints of $s^{*}$ (determined by dir), linking the leaves when necessary as it goes along. This process is guaranteed to reach the targeted endpoint of $s^{*}$, since each of the trees traversed are all rooted at $s^{*}$.

The manner in which a leaf is linked depends on the labeling of the segment of which it is an endpoint. Let $a$ be the current leaf in the sequence, belonging to component $G_{a}$, and let $b$ be the next leaf in the sequence. Let $s_{a}$ and $s_{b}$ be the segments of which $a$ and $b$ are endpoints, respectively. If $s_{b}$ is unattached, ATTACHTO introduces a bridge between 
$a$ and $b$, integrates $s_{b}$ into $G_{a}$ by assigning it the same label as $s_{a}$, and then continues the procedure from $b$.

If $s_{b}$ is not unattached, then it belongs to some connected component $G_{b}$. If $G_{b}=G_{a}$, then instead of bridging from $a$ to $b$ (and introducing an unwanted cycle into $G_{b}=G_{a}$ ), the procedure simply proceeds onward from $b$ without creating a bridge.

If $G_{b} \neq G_{a}$, then the introduction of a bridge from $a$ to $b$ forces the two components to be merged. If $G_{b}=G$, then all segments of $G_{a}$ are immediately relabeled to that of $G$, namely attached. Similarly if $G_{b}=G$, all segments of $G_{b}$ are immediately relabeled to attached. If neither $G_{b}$ nor $G_{a}$ equals $G$, then the two components are merged. In all three cases the procedure continues from $b$.

Whenever two components other than $G$ are to be merged, it would be inefficient to relabel the segments of one component explicitly to match that of the other; if this is done, a given edge could potentially be relabeled many times. Instead, an efficient set union-find data structure $U$ is used to keep track of equivalence classes of segment labels. Merging components is thus a matter of merging classes of labels. The explicit relabeling that occurs when a component is merged with $G$ can only be done once per edge - once a segment receives the label attached, its label will never change again.

The procedure by which the leaves of neighbouring extension trees are linked finishes with the initial leaf $x$ and $s^{*}$ in the same connected component; several components may have been merged with each other or into $G$ in the process. Once segment $s^{*}$ has been attached (say at its endpoint $\lambda$ ), ATTACHTO is called again starting from $\lambda$. To avoid creating two bridges at $\lambda$ in the same cell of $\mathrm{Q}$, the direction of the linking is reversed. For example, if the call ATtACHTO $(x$, left $)$ resulted in $s^{*}$ being linked to $x$, then the call $\operatorname{ATTACHTO}(\lambda$, right) would be performed.

If $\lambda$ itself is the last extension tree leaf, then by attaching $\lambda, s^{*}$ is attached. In this case, since extension tree $T_{\lambda}$ is rooted at the previously visited segment $s^{*}$, no further call to ATTACHTO is made from $\lambda$.

Figure 5 illustrates the process by which segments are attached by showing the bridges created as a result of a call to $\operatorname{ATTACHTO}(x$, right $)$, assuming the prior creation of bridge $b$. Note that in this example, the sequence of calls to ATTACHTo terminates at a node $y$ which is simultaneously the target endpoint of its segment, and the last of the leaves of the extension trees rooted at its segment.

In the case where $T_{x}$ is not rooted at a segment of $S$, but instead is rooted at the bounding box, the behaviour of ATTACHTO is somewhat different. If ATTACHTO is called when all segments are yet unattached, the circular nature of the list of neighbours results in the connection of the entire list. Once the starting point is reached, the process terminates. For an example of how ATTACHTO handles this special case, see Fig. 6.

\section{$\operatorname{ATtachTo}(x, d i r)$}

(1) If $x$ has already been marked examined, then return. Otherwise, mark $x$ as being examined.

(2) Let $T_{x}$ be the extension tree of which $x$ is a leaf, and let $r_{x}$ be the root of $T_{x}$. Let $s^{*}$ be the segment at which $T_{x}$ is rooted.

(3) If $s^{*}$ is attached or semi-attached, then return. 


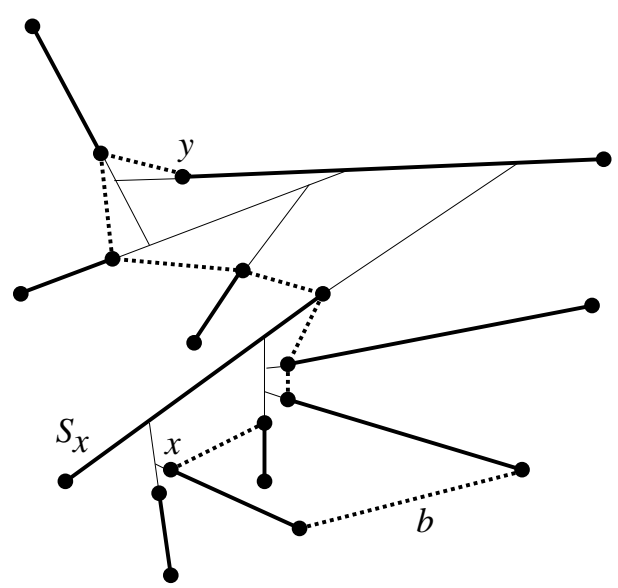

Fig. 5. Bridges created by $\operatorname{AttachTo}(x$, right $)$.

(4) ( $s^{*}$ must be an unattached segment.)

If $x$ has no neighbouring leaf in the direction $d i r$, then:

(4a) Let $w$ be that endpoint of $s^{*}$ which lies in direction dir from $r_{x}$ as viewed from $x$. If $x=w$, then return.

(4b) Otherwise:

(4b1) Create a bridge between $x$ and $w$. Mark $s^{*}$ with the label of $s_{x}$. Mark the endpoint of $s^{*}$ opposite to $w$ as pending.

(4b2) Let oppdir be the direction opposite to dir. ATTACHTO $(w$, oppdir $)$.

(5) Else, $x$ has a neighbouring leaf $y$ in the direction dir. Let $s_{y}$ be the segment of which $y$ is an endpoint.

(5a) If $s_{y}$ is unattached, then create a bridge between $x$ and $y$. Mark $s_{y}$ with the label of $s_{x}$, and the endpoint of $s_{y}$ opposite to $y$ as pending.

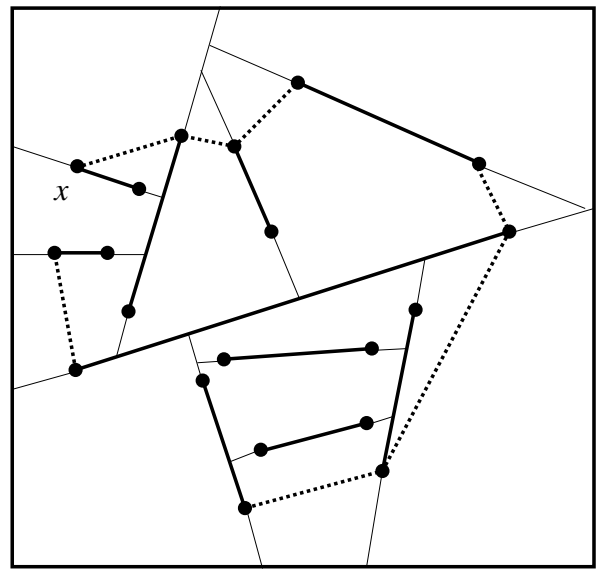

Fig. 6. Bridges created by $\operatorname{AtTACHTO}(x$, right $)$. 
(5b) Otherwise, $s_{y}$ is attached or semi-attached. If the component of $s_{y}$ is different to that of $s_{x}$, then:

(5b1) Create a bridge between $x$ and $y$.

(5b2) If $s_{x}$ is attached, then relabel all segments of the connected component containing $s_{y}$ as attached.

(5b3) Otherwise, if $s_{y}$ is attached, then relabel all segments of the connected component containing $s_{x}$ as attached.

(5b4) Otherwise, both $s_{x}$ and $s_{y}$ are semi-attached. Merge the components containing $s_{x}$ and $s_{y}$, by making their labels equivalent to each other within the union-find structure $\mathrm{U}$.

(5c) $\operatorname{ATTACHTO}(y, d i r)$.

It should be noted at this point that ATTACHTO maintains each of the invariants listed in Section 4.1. In particular, the introduction of the bridge at Step (5b1) does not violate the invariant relating to the number of bridges that may be attached at a particular endpoint: if $y$ already had a bridge attached to it, the endpoint would have had the label examinedin which case the procedure ATTACHTO would have been called at $y$ before, that would have resulted in $s^{*}$ already having been labeled attached or semi-attached. A formal inductive proof of correctness is given later in the paper.

\subsection{Stitching Up Cells}

Procedure ATTACHTO is not in itself sufficient to link all segments into a tree of degree 3. Even if ATTACHTO is applied such that no more endpoints are pending, some segments may still be unattached, and some cells of Q may not yet be connected (see Fig. 7 for an example). In these situations where ATTACHTO cannot be applied, the procedure to be outlined in this subsection takes over. Since this procedure, STITCHUP, relies heavily upon special properties of subdivisions for which ATTACHTO cannot be applied, we describe STITCHUP without worrying about its applicability at this stage. Its applicability and usefulness will be established after the overall algorithm has been described.

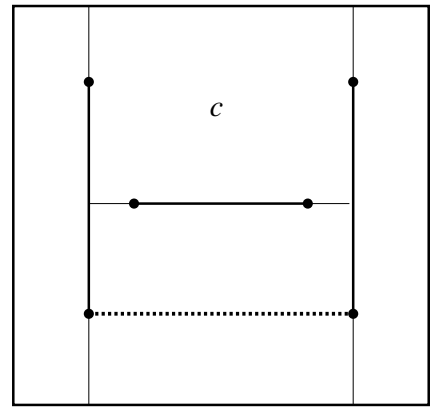

Fig. 7. No more endpoints are pending, but $c$ is not connected. 
Procedure STITCHUP is called upon cells that are labeled pending: to be precise, those that have endpoints of both attached and unattached segments in their boundaries. If $c$ is such a cell, the effect of calling STITCHUP is to attach all unattached segments having one or both endpoints lying on the boundary of $c$.

This is done in three phases. In the first phase a clockwise scan is performed around the boundary of $c$, starting from an endpoint $w_{0}$ that is guaranteed to belong to an attached segment. When the scan encounters an unattached segment with both endpoints on the boundary of $c$, it initiates a call to ATTACHTO in one of two ways, depending on the number of consecutive unattached segments encountered leading up to the current segment.

Once the first invocation of ATTACHTO has terminated, a number of previously unvisited endpoints may become pending. Calls to ATTACHTO are then initiated from each pending endpoint, until no further pending endpoints remain. The result of this process is the creation of a connected component consisting of semi-attached segments and bridges. The scan then progresses to the next segment with both endpoints in the boundary of $c$, and the process is repeated to yield another connected component.

It is shown later that when this scan has terminated, each of the previously unattached segments on the boundary of $c$ will have been integrated into a connected component. As a result of the action of ATTACHTO, some components may have merged with each other, or even with the original component of attached edges. Furthermore, every surviving semiattached component shall be shown to contain at least two endpoints on the boundary of $c$ that are not incident to any bridges passing through $c$.

In the second phase of the STITCHUP procedure, a clockwise scan is again performed, this time to identify endpoints of semi-attached components not incident to bridges through $c$. When two consecutive such endpoints are discovered from different semiattached components, a bridge is introduced, thereby merging the components. Once the scan is complete, a single semi-attached component remains.

In the final phase the remaining semi-attached component (call it $G^{\prime}$ ) is integrated into the attached component $G$, in one of two ways. If the endpoint $w_{0}$ is incident to no bridge passing through $c$, then $w_{0}$ may safely be bridged to either of two endpoints of $G^{\prime}$ (call them $\lambda$ and $\rho$ ) identified as incident to no bridges passing through $c$. Otherwise, if a bridge through $c$ is incident to $w_{0}$, it is replaced by two new bridges connecting $G$ to $G^{\prime}$. The connectivity of the endpoints of the deleted bridge is in a sense "diverted" through the new bridges and segments of the semi-attached component.

Once STITCHUp has terminated for cell $c$, all segments are again labeled either attached or unattached, and again no endpoint of a segment of $S$ is left with a label of pending. We claim, and prove later, that all segments on the boundary of $c$ that were previously unattached have become attached as a result of the call to STITCHUP.

\section{$\operatorname{STITCHUP}(c)$}

(1) Let $W=\left\{w_{0}, w_{1}, w_{2}, \ldots, w_{k-1}, w_{k}\right\}$ be the sequence of segment endpoints encountered as one traverses the boundary of $c$ in clockwise order, where $w_{0}=w_{k}$ is the first endpoint on the boundary of $c$ to have been marked by the algorithm as examined. Let $s_{i}$ be the segment of which $w_{i}$ is an endpoint, for all $0 \leq i$ $\leq k$. 
(2) Initialize the union-find structure $U$ to recognize equivalence classes within the set of labels $\{1, \ldots, k-1\}$. Each label is initially in its own equivalence class.

(3) For $i \longleftarrow 0$ to $k-1$, do the following:

(3a) If segment $s_{i}$ is attached or semi-attached, then set $a \longleftarrow i$. (a stores the most recently encountered attached or semi-attached endpoint.)

(3b) If $s_{i}$ is unattached and $s_{i}=s_{i+1}$, then:

(3b1) ( $s_{i}$ has both endpoints on the boundary of $c$.)

Mark $s_{i}$ as semi-attached with component label $i$-segment $s_{i}$ is the first in a new connected component. Mark $w_{i}$ and $w_{i+1}$ as pending.

(3b2) If $i-a$ is even, then initiate $\operatorname{ATTACHTO}\left(w_{i}\right.$, right).

(3b3) If $i-a$ is odd, then initiate $\operatorname{ATTACHTO}\left(w_{i+1}, l e f t\right)$.

(3b4) While there are endpoints yet pending, choose such an endpoint (call it $y$ ), and initiate $\operatorname{ATTACHTO}(y$, right $)$.

(4) Set $\lambda \longleftarrow \rho \longleftarrow \varnothing$.

(5) For $i \longleftarrow 0$ to $k-1$, do the following. If $s_{i}$ is semi-attached and $w_{i}$ has no bridge attached to it passing through cell $c$, then:

(5a) If $\lambda=\emptyset$, then set $\lambda \longleftarrow i$.

(5b) Otherwise, if $\rho=\emptyset$, then set $\rho \longleftarrow i$.

(5c) Otherwise, if $s_{i}$ and $s_{\rho}$ are in different semi-attached connected components, then:

(5c1) Introduce a bridge between $s_{\rho}$ and $s_{i}$ through $c$.

$(5 \mathrm{c} 2)$ Using the union-find structure $\mathrm{U}$, merge the components containing $s_{\rho}$ and $s_{i}$.

(5c3) Set $\rho \longleftarrow i$.

(5d) Otherwise, if $s_{i}$ and $s_{\rho}$ are in the same semi-attached connected component, then set $\rho \longleftarrow i$.

(6) At this point, all segments with endpoints on the boundary of $c$ are either attached, or belong to a common semi-attached connected component.

(6a) If there is no bridge attached to $w_{0}$ passing through $c$, then introduce the new bridge $\left(w_{0}, w_{\lambda}\right)$.

(6b) If there previously existed a bridge between $w_{0}$ and $w_{1}$, then delete the bridge and replace it with bridges $\left(w_{1}, w_{\lambda}\right)$ and $\left(w_{\rho}, w_{0}\right)$.

(6c) Otherwise, there previously existed a bridge between $w_{0}$ and $w_{k-1}$. Delete this bridge and replace it with bridges $\left(w_{0}, w_{\lambda}\right)$ and $\left(w_{\rho}, w_{k-1}\right)$.

(7) Relabel all semi-attached segments in $S$ as being attached.

Note that STITCHUP maintains each of the invariants listed in Section 4.1; in particular, a call to STITCHUP cannot result in the connection of more than one bridge through $c$ at any endpoint of any segment. The proof of this claim follows the discussion of Algorithm ENCOMPASS in the next section.

\subsection{The Main Algorithm}

Having described procedures ATTACHTO and STITCHUP, we are now in a position to outline the main algorithm. Following this, we prove that it is correct. 


\section{ENCOMPASS}

(1) From the set of segments $S$, build its associated convex subdivision using the method outlined in Section 2.

(2) Mark the bounding box and each segment as unattached. Mark each segment endpoint and each cell unvisited.

(3) Connect the leaves of all trees rooted at the bounding box, as follows:

(3a) Choose such a leaf (call it $x$ ). Let $s_{x}$ be the segment of which $x$ is an endpoint.

(3b) Mark $s_{x}$ as attached, and the endpoint of $s_{x}$ opposite to $x$ as pending.

(3c) $\operatorname{ATTACHTO}(x$, right).

(3d) Mark the bounding box as attached.

(4) While there are endpoints yet pending do:

(4a) Choose such an endpoint (call it $y$ ).

(4b) $\operatorname{ATTACHTO}(y$, right).

(5) While there are cells yet pending do:

(5a) Choose such a cell (call it $c$ ).

(5b) $\operatorname{StitchUp}(c)$.

The proof of correctness of Algorithm ENCOMPASS is by induction. It is easily seen that the first segments are correctly attached to $G$ at Step (3) of ENCOMPASS. For each remaining segment $s$ of $S$ attached at Step (4) or Step (5), we assume inductively that both Lemmas 7 and 8 hold true before $s$ is attached, and show that both also hold after $s$ is attached.

The proofs of the lemmas rely on two main facts. First, ATTACHTo and STITCHUP both maintain the invariants set out in Section 4.1. In particular, any endpoint labeled unvisited or pending is not connected to a bridge. Both ATTACHTO and STITCHUP ensure that when a new bridge is introduced, its endpoints will have acquired the label examined. Second, any endpoint with the label examined must have been given this label by ATTACHTO.

Lemma 7. Let $s_{v}$ be a segment labeled either attached or semi-attached, and let $v$ be an endpoint of $s_{v}$ which has become examined as a result of the application of ATTACHTO upon $v$. Let $T_{v}$ be the extension tree of which $v$ is a leaf, and let $s$ be the segment of $S$ at which $T_{v}$ is rooted. If $s$ was unattached at the time that ATTACHTO was invoked on $v$, then once this invocation has terminated, s must have correctly been made a member of the same connected component as $s_{v}$.

Proof. We assume that $s$ is not in the same connected component as $s_{v}$ after the invocation of ATTACHTO on $v$ has terminated. If the call to ATTACHTO on $v$ did not immediately result in $s$ being bridged to $v$, then ATTACHTO attempted to link the neighbouring leaves of extension trees to an endpoint of $s$ by means of bridges. If all these leaves were labeled unvisited or pending, then clearly the algorithm succeeded in bridging to and attaching the segment $s$, as no bridges had previously been attached to these leaves. Therefore at least one of the leaves (call it $v^{\prime}$ ) on the path to the endpoint of $s$ must have previously been examined. As $v^{\prime}$ can only have been given this label by ATTACHTO, the induction hypothesis implies that $v^{\prime}$ and $s$ are in the same connected component. However, if $v^{\prime}$ 
and $v$ are not already in the same connected component, the bridge $\left(v, v^{\prime}\right)$ introduced at Step (5b1) of ATTACHTO causes the components to be merged. From this contradiction, the result follows.

Lemma 8. Let c be a pending cell upon which the call $\mathrm{STITCHUP}(c)$ is made, at a time when all segments of $S$ are labeled either attached or unattached. Then when the call to STITCHUP terminates:

1. All segments of $S$ are again labeled either attached or unattached.

2. No endpoints of segments of $S$ are pending.

3. $c$ is correctly connected.

Proof. Consider the sequence of segment endpoints $W=\left\{w_{0}, w_{1}, w_{2}, \ldots, w_{k-1}, w_{k}\right\}$ encountered as one traverses the boundary of $c$ in clockwise order, where $w_{0}=w_{k}$ is the first endpoint on the boundary of $c$ to have been marked by the algorithm as examined. Let $s_{i}$ be the segment of which $w_{i}$ is an endpoint, for all $0 \leq i \leq k$.

Imagine the boundary of $c$ as viewed from the interior, in clockwise order starting from $w_{0}$. The extension edge emanating from each endpoint of $W$ must itself lie on the boundary of $c$. If the extension edge of $w_{i}$ follows $w_{i}$ when scanning the boundary in clockwise order, then we say that $w_{i}$ is a clockwise $(\mathrm{CW})$ endpoint of $W$. Otherwise, $w_{i}$ is called a counterclockwise (CCW) endpoint.

We first show that after the loop of Step (3) has terminated, all segments on the boundary of $c$ are either attached or semi-attached. The invariants maintained during the execution of the loop are:

1. Immediately before the execution of Step (3b1), $s_{j}$ is attached or semi-attached for all $0 \leq j \leq a$.

2. Immediately after the execution of Step (3b4), $s_{j}$ is attached or semi-attached for all $0 \leq j \leq i+1$.

3. Immediately after the execution of Step (3b4), $s_{j}$ belongs to the same connected component as $s_{i+1}$, for all $a<j \leq i$.

4. Except during the execution of Steps (3b1)-(3b4), no endpoints of edges are pending.

5. Immediately after the execution of Step (3b4), if $s_{i}$ is labeled semi-attached, then there exist two endpoints $w_{j_{1}}$ and $w_{j_{2}}$ of $W$ such that:

- there are no bridges passing through the interior of $c$ having $w_{j_{1}}$ or $w_{j_{2}}$ as an endpoint;

- $j_{1}<j_{2} \leq i+1$;

- if $b$ is the smallest index such that $s_{b}$ belongs to the same semi-attached component as $s_{i}$, then $s_{j}$ also belongs to this component for all $b \leq j \leq j_{2}$.

Note that when STITCHUP is invoked, no endpoints can be labeled pending. Also note that in the first iteration of the loop, $a$ is set to 0 , since $s_{0}$ had been attached prior to the call to STITCHUP.

Consider now the situation at $i=p$ in which segment $s_{p}$ is found to be unattached at Step (3a), and $s_{p-1}$ has the label attached or semi-attached. Endpoint $w_{p}$ cannot be $\mathrm{CW}$; otherwise, the sequence of edges on the boundary of $c$ between $w_{p-1}$ and $w_{p}$ 
would consist of extension edges, followed by a single segment edge adjacent to $w_{p}$. The extension tree of which $w_{p-1}$ is a leaf would therefore be rooted at $s_{p}$. Since the loop invariant implies that $w_{p-1}$ cannot be labeled pending (and therefore must be examined), Lemma 7 implies that $s_{p}$ must be attached or semi-attached - a contradiction. Therefore $w_{p}$ must be CCW in this situation.

Let $p<q \leq k$ be the smallest index such that either $w_{q}$ is $\mathrm{CW}$, or $s_{q}$ is attached or semi-attached. We claim that in fact $s_{q}$ must be unattached. Otherwise, we have two cases:

- $w_{q}$ is $C W$.

From the definition of $q$, we have that $w_{q-1}$ is CCW. The only way a CCW endpoint can be followed by a CW endpoint in CW order about the boundary of $c$ is if the endpoints belong to the same segment. However, the assumption that $s_{q}$ is attached or semi-attached implies that $s_{q-1}=s_{q}$ is also attached or semi-attached. This contradicts the minimality of $q$.

- $w_{q}$ is CCW.

Since $w_{q-1}$ is also CCW, the sequence of edges on the boundary of $c$ between $w_{q-1}$ and $w_{q}$ would consist of extension edges, preceded by a single segment edge adjacent to $w_{q-1}$. The extension tree of which $w_{q}$ is a leaf would therefore be rooted at $s_{q-1}$. Since the loop invariant implies that $w_{q}$ cannot be labeled pending (and therefore must be examined), Lemma 7 implies that $s_{q-1}$ must be attached or semi-attached-again contradicting the minimality of $q$.

We are forced to conclude that $w_{q}$ is $\mathrm{CW}$, and also that $s_{q-1}=s_{q}$. This implies the following:

- Once an unattached segment $s_{p}$ is discovered at Step (3a), the condition of Step (3b) will eventually be met at some $i \geq p$.

- Segment $s_{j}$ is unattached for all $p \leq j \leq i+1$.

- Endpoint $w_{j}$ is CCW for all $p \leq j \leq i$.

When Steps (3b1)-(3b4) are executed, the effect is to render semi-attached (or possibly even attached) every segment $s_{j}$ in the range $p \leq j \leq i+1$. To prove that this is the case, consider the effect of initiating ATTACHTO at endpoint $w_{j}$, where $w_{j}$ and $w_{j-1}$ are both CCW. Arguments similar to those appearing above ensure that as a result of the call to ATTACHTO, segment $s_{j-1}$ becomes a member of the same connected component as $s_{j}$. Endpoint $w_{j-1}$ becomes either pending or examined, depending on the endpoint by which $s_{j-1}$ becomes attached. However, Step (3b4) ensures that all pending endpoints become examined before the step terminates. Given that both endpoints of $s_{i}=s_{i+1}$ are labeled pending in Step (3b1), and that all $w_{j}$ are $\mathrm{CCW}$ and $s_{j}$ are initially unattached for all $p \leq j \leq i$, all $w_{j}$ in the range must become examined by the time Step (3b4) terminates.

When Step (3b4) terminates, the second through fourth loop invariants mentioned above have been restored. Since $s_{i+1}=s_{i}$ becomes semi-attached (or possibly even attached) as a result, $a$ is set to $i+1$ in the next iteration of the loop. This guarantees that the first loop invariant holds for the next execution of Step (3b1), if any. Thus when the loop terminates, all segments in the boundary of $c$ are indeed labeled either attached or semi-attached. 
So far we have not justified the separate handling of the cases depending on the parity of $i-a$, in Steps (3b2) and (3b3). We claim that this separate handling allows the fifth loop invariant to be maintained. We assume then that $s_{i}$ is semi-attached when Step (3b4) terminates.

If $i-a$ is even, then Step (3b2) prevents $w_{i}$ from receiving a bridge through the interior of $c$ before the termination of Step (3b4)—since all bridges introduced in Step (3b) merge segments into a common connected component, a second bridge through $w_{i}$ would introduce a cycle. In order to identify a second endpoint that receives no bridge through $c$, consider the endpoints whose indices lie in the range $\{a+1, \ldots, i-1\}$. Any bridges introduced through $c$ at any of the endpoints in the range must either link two consecutive endpoints in the range, or must link $w_{a+1}$ with $w_{a}$. We have two cases:

- $w_{a+1}$ is not bridged to $w_{a}$.

Since no endpoint can receive more than one bridge, an even number of endpoints of the range must receive bridges. As the cardinality of the range is odd, there must be at least one endpoint that receives no bridge through $c$. Together with $w_{i}$, this leaves the semi-attached component of $s_{i}$ with at least two unused endpoints with indices in the range $\{a+1, \ldots, i+1\}$.

Let $b$ be the smallest index such that $s_{b}$ belongs to the same semi-attached component as $s_{i}=s_{i+1}$. If $b=a+1$, we are done, since $s_{j}$ is in the component of $s_{i}$ for all $a+1 \leq j \leq i+1$. Otherwise, $b \leq a$. Immediately before the current iteration of the loop, segment $s_{b}$ must have been the segment of smallest index of a different semi-attached component. If so, the fifth loop invariant guarantees the existence of endpoints $w_{j_{1}}$ and $w_{j_{2}}$ to which no bridges were incident through $c$ before the current iteration where $b \leq j_{1}<j_{2}$. Since $s_{j^{\prime}}$ was in the same component as $s_{b}$ for all $b \leq j^{\prime} \leq j_{2}$, then $j_{2} \leq a$. Also since no bridge was introduced at the current iteration between $w_{a}$ and $w_{a+1}$, the endpoints $w_{j_{1}}$ and $w_{j_{2}}$ are not incident to bridges through $c$ when the current iteration terminates. The fifth loop invariant is therefore satisfied in this case.

- $w_{a+1}$ is bridged to $w_{a}$.

If this occurs, the connected component of $s_{i}$ merges with that of $s_{a}$. If $s_{a}$ is attached, then $s_{i}$ becomes attached, contradicting our assumption that $s_{i}$ was semi-attached at the end of Step (3b4). If $s_{a}$ is semi-attached, then the result of the merge is a single semi-attached component. By the fifth loop invariant, at least two endpoints $w_{j_{1}}$ and $w_{j_{2}}$ of $W$ in the semi-attached component to which $s_{a}$ belonged had no bridge attached to them passing through $c$. Let $b$ be the smallest index of the segments in the component of $s_{a}$. As before, $b \leq j_{1}<j_{2} \leq a$.

If $j_{2}<a$, then the arguments of the previous case apply to show that the fifth loop invariant continues to hold. Otherwise, $j_{2}=a$. Since $s_{j}$ belongs to the former component of $s_{a}$ for all $b \leq j \leq a$, and to the component of $s_{i}$ for all $a+1 \leq j \leq i$, after the merge, $s_{j}$ belongs to the component of $s_{i}$ for all $b \leq j \leq i+1$. Endpoint $w_{j_{1}}$ cannot have received a bridge as a result of the merge, and therefore $w_{j_{1}}$ and $w_{i}$ satisfy the conditions of the fifth loop invariant.

If $i-a$ is odd, Step (3b3) ensures that $w_{i+1}$ will have no bridge attached to it through $c$. Considering that the range of indices $\{a+1, \ldots, i\}$ is of odd cardinality, the fifth loop 
invariant can be shown to hold using an argument almost identical to that of the case where $i-a$ is even.

At this point, we have shown that all five invariants are maintained by the loop of Step (3). In particular, when the loop terminates, the second, third, and fifth invariants still hold, and no endpoints of segments in $S$ are pending. Each bridge passing through $c$ must link consecutive endpoints of $W$, since it can only have been introduced via a call to ATTACHTO. Such bridges cannot interfere with any other bridges that may later be introduced between free endpoints of $W$.

The loop in Step (5) uses the index $\rho$ to maintain the most recently encountered unbridged endpoint of the current semi-attached component; whenever an unbridged endpoint of a new component is discovered, a bridge is introduced between $w_{\rho}$ and the new endpoint, merging the components. The fifth invariant guarantees that when the first unbridged endpoint $w_{j_{1}}$ of a new component is discovered, a second unbridged endpoint $w_{j_{2}}$ of that component also exists, with $j_{2}>j_{1}$. This ensures that the merged component has an unbridged endpoint that can be used to merge the next component to be discovered by the loop of Step (5). When the loop terminates, all semi-attached edges have been merged into one component, and $\lambda$ and $\rho$ are the minimum and maximum indices of the original unbridged endpoints taken over all semi-attached segments.

In Step (6) the semi-attached component is merged into the attached component via endpoints $w_{0}, w_{\lambda}$, and (perhaps) $w_{\rho}$. Since there exists no $j$ in the interval $\{0, \ldots, \lambda\}$ such that $w_{j}$ was an unbridged endpoint of a semi-attached segment before Step (5), the bridges $\left(w_{0}, w_{\lambda}\right)$ would intersect no other bridges through $c$ if introduced; similarly, the bridges $\left(w_{1}, w_{\lambda}\right)$ and $\left(w_{\rho}, w_{k-1}\right)$ would intersect no other bridges.

If $w_{0}$ is not already incident to a bridge through $c$, the introduction of bridge $\left(w_{0}, w_{\lambda}\right)$ correctly merges the semi-attached and attached components. Otherwise, if the bridge $\left(w_{0}, w_{1}\right)$ exists, replacing $\left(w_{0}, w_{1}\right)$ by $\left(w_{1}, w_{\lambda}\right)$ and $\left(w_{\rho}, w_{0}\right)$ correctly splices the semiattached component into the attached component between $w_{0}$ and $w_{1}$. The result is a single connected component that includes all endpoints of $W$.

To conclude the proof, we note that when STITCHUP has terminated, no segments are semi-attached and no endpoints are pending.

Lemma 9. ENCOMPASS eventually terminates after taking at most $O(n \log n)$ time.

Proof. The construction of the underlying convex subdivision Q can be accomplished using planar line-sweep techniques, as outlined in Section 2, in $O(n \log n)$ time [11]. At the time of construction, pointers can be established linking the leaves of extension trees with the segments to which they are rooted, and counters can be set up to allow efficient modification of the labels of cells.

The total amount of work done in executing procedure ATTACHTO is proportional to the number of vertices and edges of $\mathrm{Q}$, which by Lemma 1 is in $O(n)$. The first time ATTACHTO is called on an endpoint, it marks it as examined; if called on the endpoint again, it simply exits without doing anything (this can be charged to the neighbour from which the call was made). The extension edges of $Q$ can be traversed at most once in each direction when moving from neighbour to neighbour; segment edges can be traversed at most four times each (twice from each of the two cells it bounds). 
The total work done in executing STITCHUP on $c$ can be divided into three categories: work involving the union-find structure U; work involving calls to ATTACHTO; and the remainder of the work. The work involving calls to ATTACHTO has already been accounted for. Also, the relabeling of the segments in the final step of STITCHUP can be charged to the segments themselves-since each segment can become attached only once, the total work performed in this step over all calls to STITCHUP is in $O(n)$.

Let $k_{c}$ be the number of subdivision edges on the boundary of cell $c$; this number is larger than the number of segment endpoints on the boundary. The total number of union-find operations is in $O\left(k_{c}\right)$, as well as the total time taken which has not already been accounted for by calls to ATTACHTO, or the relabeling discussed above. If standard union-find structures are used [4], the time taken to perform these operations is in $O\left(k_{c} \cdot \alpha\left(k_{c}\right)\right.$ ), where $\alpha\left(k_{c}\right)$ is the very slowly growing inverse of Ackermann's function. Since STITCHUP can only be performed once per cell, the total time taken by calls to STITCHUP is in $O\left(\sum_{c}\left(k_{c} \cdot \alpha\left(k_{c}\right)\right)\right) \subseteq O\left(\alpha(n) \sum_{c} k_{c}\right)$. Since each non-box edge of the subdivision is contained in exactly two cells, and since the number of cells is in $O(n), \sum_{c} k_{c}$ is proportional to the total number of edges of $\mathrm{Q}$, which is in $O(n)$. The total additional time taken by the calls to STITCHUP is therefore in $O(n \cdot \alpha(n))$.

The overall work performed by ENCOMPASS is accounted for by the total work involved in calls to ATTACHTO, to the additional work performed by STITCHUP, and to the construction of the convex subdivision. The time taken to construct the subdivision dominates, and thus the total time taken by ENCOMPASS is in $O(n \log n)$.

Lemma 10. When the execution of ENCOMPASS terminates, then $G$ is a degree-3 encompassing tree for $S$.

Proof. Lemma 9 implies that ENCOMPASS does indeed terminate.

Assume that $G$ does not encompass all segments of $S$. Then there exists at least one cell of Q that is unvisited. Since no cell is pending (by Step (5)), all cells that are not unvisited must be connected. The correctness of Step (3) ensures that all cells bordering the bounding box are not unvisited; therefore, they must all be connected.

Let $\mathrm{C}$ be the union of all cells that are unvisited. The boundary of $\mathrm{C}$ consists of a collection of disjoint simple cycles in Q. There is at least one cycle in the boundary; call it $C$. Since $C$ cannot contain box segments, Lemma 2 implies that there exists some segment endpoint $v$ on $C$. Since $v$ is on the boundary of an unvisited cell, $v$ must be labeled unvisited. However, $v$ is also on the boundary of a connected cell, and must therefore be labeled examined - a contradiction. Every cell must therefore be connected. Lemma 6 then implies that $G$ encompasses all segments of $S$.

The planarity and degree of $G$ are a result of the invariants set forth in Section 4.1 .

Theorem 11. Given a set $S$ of $n$ disjoint line segments in the plane, ENCOMPASS computes a degree-3 planar encompassing tree of $S$ in $O(n \log n)$ time and $O(n)$ space.

Proof. Follows from Lemmas 1 and 7-10. 


\section{Lower Bound}

Finally, we show that the problem of finding a degree-3 encompassing tree of a set of disjoint line segments requires $\Omega(n \log n)$ time to solve, using a reduction similar to that for the convex hull problem [11]. This implies the optimality of Algorithm ENCOMPASS.

Theorem 12. The problem of sorting $n$ real numbers is $O(n)$-transformable to the problem of finding a degree-3 spanning tree of a set of disjoint line segments; thus, finding a degree-3 encompassing tree of a set of disjoint line segments requires $\Omega(n \log n)$ time.

Proof. Given a set $S$ of $n$ positive real numbers, $x_{1}, \ldots, x_{n}$, we show how any encompassing tree algorithm can be used to sort them with only linear overhead. For convenience, let $s_{1}, \ldots, s_{n}$ represent the indices of the sorted order of the real numbers from smallest to largest; that is, $x_{s_{1}}$ is the smallest of the numbers, and $x_{s_{n}}$ is the largest. Let $m=x_{S_{n}}$ be the maximum element of $S$.

For each number $x_{i}$, we construct a corresponding vertical line segment, $l_{i}$, i.e., we associate the number $i$ with the line segment. The line segment $l_{i}$ is constructed in the following way. The lower endpoint has coordinates $\left(x_{i}, x_{i}^{2}-m^{2}-1\right)$, and the upper endpoint has coordinates $\left(x_{i},-x_{i}^{2}+m^{2}+1\right)$. Note that since $m$ can be computed in linear time, the construction requires only linear time.

These endpoints are well defined-for all values of $i$, the lower endpoint is strictly below the $x$-axis, while the upper endpoint is strictly above. All of the lower endpoints lie on the upward-opening parabola $L: y=x^{2}-m^{2}-1$ and all of the upper endpoints lie on the downward-opening parabola $U: y=-x^{2}+m^{2}+1$. Since the endpoints are on the boundary of the a convex region (namely that bounded by $U, L$, and the $y$-axis), the fact that the segments are parallel means that the endpoints of $l_{s_{i}}$ are visible from the endpoints of no other edges except $l_{s_{i-1}}$ and $l_{s_{i+1}}$.

Since the degree- 3 encompassing tree consists of visibility edges between line segments together with the line segments themselves, a simple depth-first traversal of the tree starting from the leftmost vertex of degree 1 enables us to uncover the sorted order of the input in linear time from the output delivered by any algorithm.

Although the segments of the proof were chosen to be parallel for the sake of convenience, constructions in which no two segments are parallel can also be used.

\section{Conclusion}

In this paper we have shown that a set of disjoint line segments always admits an encompassing tree with maximum vertex degree 3 , and that there exist configurations of line segments such that any encompassing tree of the set has maximum degree 3 . We presented an algorithm to compute a binary encompassing tree in $O(n \log n)$ time, and 
showed a lower bound of $\Omega(n \log n)$ for the problem establishing the optimality of our algorithm. There are a number of open problems still to be considered.

1. Is it NP-hard to compute a simple polygon or a simple hamiltonian path through a set of disjoint line segments? Rappaport [12] has shown that the decision problem is NP-complete when the line segments are allowed to intersect at their endpoints.

2. Is it possible to compute a simple polygon through a set of disjoint line segments, where the line segments are either part of the boundary, internal diagonals, or external diagonals [7]? Urabe and Watanabe [17] have shown that if the line segments are limited to the boundary and internal diagonals, that it is not always possible.

3. Is the visibility graph of a set of disjoint line segments hamiltonian [7]? If not, can anything be said about the longest path in the visibility graph?

\section{Acknowledgments}

We thank Hossam ElGindy, Eduardo Rivera-Campo, and Jorge Urrutia for fruitful discussions on the topic. We thank David Thompson for providing a stimulating research environment.

\section{References}

1. D. Avis and D. Rappaport, Computing monotone simple circuits in the plane, in Computational Morphology, G. T. Toussaint (ed.), Elsevier Science/North-Holland, Amsterdam, 1988.

2. J. A. Bondy and U. S. R. Murty, Graph Theory with Applications, Elsevier Science, New York, 1976.

3. P. Bose and G. Toussaint, Growing a tree from its branches, Journal of Algorithms 19 (1995), 86-103.

4. T. H. Cormen, C. E. Leiserson, and R. L. Rivest, Introduction to Algorithms, MIT Press, Cambridge, MA, 1990.

5. H. ElGindy and G. Toussaint, Efficient algorithms for inserting and deleting edges from triangulations, in Proc. International Conference on Foundations of Data Organization, Kyoto, 1985, pp. 163-169.

6. B. Grünbaum, Hamiltonian polygons and polyhedra, Geombinatorics 3 (1994), 83-89.

7. A. Mirzaian, Hamiltonian triangulations and circumscribing polygons of disjoint line segments, Сотриtational Geometry: Theory and Applications 2 (1992), 15-30.

8. C. Monma and S. Suri, Transitions in geometric minimum spanning trees, Discrete \& Computational Geometry 8 (1992), 265-293.

9. J. O’Rourke, Art Gallery Theorems and Algorithms, Oxford University Press, New York, 1987.

10. J. O'Rourke and J. Rippel, Two segment classes with Hamiltonian visibility graphs, Computational Geometry: Theory and Applications 4 (1994), 209-218.

11. F. Preparata and M. Shamos, Computational Geometry: An Introduction, Springer-Verlag, New York, 1985.

12. D. Rappaport, Computing simple circuits from a set of line segments is NP-complete, in Proc. 3 rd ACM Symposium on Computational Geometry, Waterloo, Ontario, 1987, pp. 322-330.

13. D. Rappaport, H. Imai, and G. T. Toussaint, Computing simple circuits from a set of line segments, Discrete \& Computational Geometry 5 (1990), 289-304.

14. E. Rivera-Campo and J. Urrutia, Personal communication, 1992.

15. X. Shen and H. Edelsbrunner, A tight lower bound on the size of visibility graphs, Information Processing Letters 26 (1987), 61-64. 
16. T. Su and R. Chang, Computing the constrained relative neighborhood graphs and constrained Gabriel graphs in Euclidean plane, Pattern Recognition 24 (1991), 221-230.

17. M. Urabe and M. Watanabe, On a counterexample to a conjecture of Mirzaian, Computational Geometry: Theory and Applications 2 (1992), 51-53.

18. A. Yao, An $O(E \log \log V)$ algorithm for finding minimum spanning trees, Information Processing Letters 4 (1975), 21-23.

Received September 14, 1999, and in revised form January 17, 2001. Online publication August 29, 2001. 